\title{
Meet the editor series—Massimo Vassalli
}

\author{
Massimo Vassalli ${ }^{1}$ iD
}

Accepted: 12 January 2021 / Published online: 1 February 2021

(C) International Union for Pure and Applied Biophysics (IUPAB) and Springer-Verlag GmbH Germany, part of Springer Nature 2021

It is my pleasure to write a few words to introduce myself to the readers of Biophysical Reviews as part of the 'meet the editors' series.

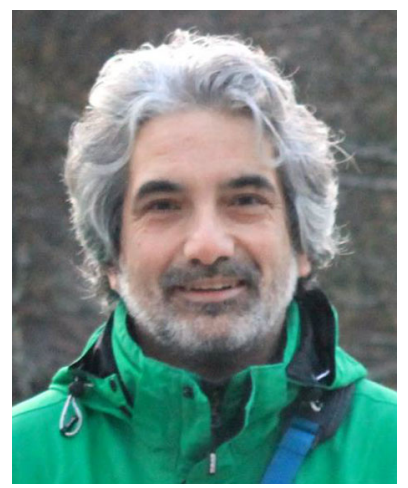

\section{University studies and training}

I started my scientific journey in the early 1990 s, at the University of Florence (Italy) where I enrolled in the Physics course. Like for many colleagues, the main trigger to select this particular pathway was the fascinating mystery of the universe - with additional inspiration provided by the world renown Arcetri Astrophysical Observatory where Galileo Galilei worked at the beginning of the seventeenth century (Righini 1967). During 1995, I moved to the University of Mainz (Germany) under the Erasmus exchange program, and I was deeply impressed by the celebrations associated with the award of the Nobel Prize to Prof Paul J. Crutzen who, at that time, was affiliated to the Max-Planck-Institut für Chemie of Mainz (Crutzen 1995). Stimulated by those

Massimo Vassalli

Massimo.Vassalli@glasgow.ac.uk

1 James Watt School of Engineering, University of Glasgow, Center for the Cellular Microenvironment, 70 University Avenue, School of Engineering, G12 8LT, Glasgow, UK events, I decided to move my focus towards a more applied field of Physics, and in my curriculum, I integrated courses about light-matter interaction and solid-state physics. Once back in Florence, I started looking for a final project supervisor interested in the mechanical properties of solids. During my quest, I was directed to the National Institute of Optics, where I met Dr. Antonio Politi. It was there that I decided to conduct, under his supervision, a numerical study on heat transport and chaos in solids (Giardina 2000; Giacomelli et al. 2000). Antonio is no longer in Florence- he is currently the Director of the Institute of Pure and Applied Mathematics (IPAM) in Aberdeen (UK). Although I slowly diverged from theoretical Physics, I still remember the nights I spent programming the Cray T3E supercomputer (Zanghirati et al. 1999) using the first Fortran implementation of the Message Passing Interface (MPI) parallel calculus library (Clarke et al. 1994). The very last point of my simulation took a record time of 26 days to be evaluated!

\section{PhD study and the move to biophysics}

After my degree in Physics, I initially moved to industry, but after 2 years, I realized I would better enjoy an academic career, where investigation and curiosity-driven discovery is the main driving force. To fulfil this vision, I enrolled in the multidisciplinary PhD course 'Non-linear Dynamics and Complex Systems' organized at the Engineering faculty of the University of Florence. The teaching committee of this doctoral course was composed by an incredibly diverse and multidisciplinary group of professors, adopting statistical mechanics and physical tools to the study of several applied problems. In this scientifically vibrant context, I met my PhD mentors, Franco Quercioli and Bruno Tiribilli. They were setting up the Biophotonics Laboratory of the National Research Council, strategically located within the main Florence hospital, and I was fascinated by the possibility of applying my technical and theoretical skills to the study of biological systems. This is the reason why I accepted a funded scholarship within the Biophotonics Lab, to design and 
develop an Atomic Force Microscope (AFM) optimized for the study of the nanomechanics of single molecules. Thanks to the expertise in optics and microscopy of Franco and Bruno, I learnt how to develop an AFM from scratch, and together, we developed new imaging modes (Pini et al. 2010; Paoletti et al. 2011; Vassalli et al. 2010; Paoletti et al. 2011; Michele Basso et al. 2011) and exploited AFM cantilevers for sensing applications (Papi et al. 2008, 2006; M. Basso et al. 2008).

In this context, I developed a peculiar interest for using the AFM as a tool for measuring mechanical properties at the nanoscale. The AFM cantilever behaves almost like an ideal spring, and it enables the measurement of interaction (mechanical) forces with an unprecedented resolution. This is particularly interesting for the study of protein folding (Best and Clarke 2002). A few years before the start of my $\mathrm{PhD}$, the possibility to stretch single molecules with the AFM was demonstrated in a seminal paper in Science (Rief 1997). Exploiting this approach, it is possible to measure the free energy landscape of a single molecule, but to obtain a correct representation, it is mandatory to consider the inherent out-of-equilibrium nature of the AFM-based mechanical unfolding process. This aspect was first addressed by Bustamante and co-workers (Liphardt 2002) who adopted an emerging theoretical framework connecting out-of-equilibrium measurements to equilibrium thermodynamics by means of a weighted average (Crooks 1999; Jarzynski 1997). We extended this approach to study a multimeric mechanical protein, and we obtained the first reconstruction of the free energy landscape of the core 8-mer of the protein Titin (Imparato et al. 2008; Marchetti et al. 2011).

After my $\mathrm{PhD}$ studies, I was granted a permanent position as Researcher within the Florence quarter of the Complex Systems Institute, belonging to the largest Italian research institution, the National Research Council (CNR). I continued my research activity on the characterization of single molecule mechanics in the context of the Nanoscopy Lab. I studied many intriguing biological systems, but a special mention should go to elastomeric proteins (Tatham and Shewry 2000). These proteins behave very much like ideal springs and constitute the backbone of the mechanical machinery of our organism. Among all proteins belonging to this class, the champion for humans is undoubtedly elastin, while insects can offer additional inspiration such as spider silk or resilin, which provides grasshoppers with their incredible jumping ability. We adopted AFM-based single molecule stretching to characterize the mechanical behaviour of elastinlike peptides (Vassalli et al. 2013; Francesca Sbrana et al. 2011) and a chimera including selected sequences from elastin resilin and collagen (Vassalli et al. 2013; Francesca Sbrana et al. 2012). The ability of these biomimetic molecules to demonstrate quasi-ideal elasticity is astonishing, and it highlights the importance of physical concepts for biological systems. This consideration led to my final move into the world of biophysics that I like to define as the study of the physical processes that occur within living organisms.

\section{From molecular to cellular biophysics}

During 2009, I moved to the Institute of Biophysics (IBF) of the CNR in Genova to establish the NanoBioScience Laboratory. IBF has a long-lasting tradition of electrophysiology, and the focus of the researchers of the institute is clearly oriented towards the study of membrane molecules within their physiological environment, the cell. The institute provides all the required facilities to perform a comprehensive biophysical study, from molecular biology and protein purification to cell culture and a small animal facility, offering both mice and cold blood animals (such as Xenopus laevis and Ambystoma mexicanum). Immersed in this new cultural environment, I gradually moved from the observation of purified molecules in a completely controlled environment to the study of proteins integrated in their physiological environment, the cell.

Collaborating with biomedical groups at the University of Genova, I made an interesting journey through neuroscience, contributing with high-resolution AFM imaging and single molecule force spectroscopy. We studied the aggregation and misfolding of several key proteins, such as synapsin, central in neuronal functionality (Monaldi et al. 2010); the APP and the A $\beta$ peptides, involved in the Alzheimer's disease (Tsushima et al. 2015; Galante et al. 2012); or the cellular prion, associated to transmissible encephalopathy (Thellung et al. 2013). Exploiting novel advances in single cell force spectroscopy (Helenius et al. 2008), we further extended the investigation to include cell-cell and cell-substrate interaction, trying to identify a single cell mechanical indicator correlated to the invasiveness of different neuroblastoma cells (Mescola et al. 2012; Gavazzo et al. 2013).

This concept of 'physical phenotype' of cells has been the leading motivation for many projects in the years to come. We investigated several patho-physiological systems, looking for specific label-free biomarkers suitable to describe the state of the biological system. We successfully applied the approach to mesenchymal stem cells along the differentiation pathway (Petecchia et al. 2015; Bloise et al. 2019), to hepatocytes under different steatogenic hits (Baldini et al. 2019), and to the maturation of osteoblasts (Bartolozzi et al. 2020).

\section{Mechanobiology}

The expertise for measuring forces at the nanoscale, the physical perspective in the investigation and the interest in cell physiology, made me rapidly converge towards the emerging field of mechanobiology, where the cell is described as a machine and mechanical concepts govern its integrity (Sheetz and Hanry 2018). In this context, the legacy of electrophysiology present at the Institute of Biophysics drove my attention to the recently discovered mammalian mechanosensitive ion channels Piezo1 and Piezo2 (Coste et al. 2010). I completely fell in love with this incredible molecular machinery, 
organized as a trimer in the plasma membrane with a unique triskelion-like structure and being able to sense mechanical stimuli with an exquisite sensitivity. To learn as much as possible about this molecule, in 2016, I exploited an Endeavour Fellowship to relocate for a period to the Victor Chang Cardiac Research Institute in Sydney, working in the laboratory of Boris Martinac. Besides being a world expert in the study of mechanosensitive ion channels, Boris is animated by a rare enthusiasm and a sincere passion for science. During my stay in Australia, we studied the role of cholesterol in the organization and functionality of Piezo1 (Ridone et al. 2020; Ridone et al. 2019). Moreover, this experience was a real turn point in my career, and it contributed to consolidation of the idea that mechanobiology is something I would like to be deeply involved in.

I have ever been active within the European biophysics' community, serving as secretary of the Italian Society for Pure and Applied Biophysics from 2014 to 2016 and from 2018 to present and actively participating to the Regional Biophysics Conferences and to the European Biophysical Societies Associations congresses. Nevertheless, a proper interdisciplinary event focused on mechanobiology was missing at that time. This is what we realized, with colleague and friend Aldo Ferrari, when we decided to establish the series of conferences Nanoengineering for Mechanobiology, organized together every year since 2016 (Ferrari et al. 2019). This conference is nowadays a tradition, and it constitutes a place where bioengineers, biophysicists, biologists, and medical researchers can meet and discuss the role of mechanobiology in physiology and pathology.

At the end of 2019, I was appointed Reader at the School of Engineering of the University of Glasgow, and I had the opportunity to move within the Centre for the Cellular Microenvironment led by Manuel Salmeron-Sanchez and Matthew Dalby. This environment offers the possibility to pursue impact-driven research, in close interaction with industrial actors and biomedical stakeholders in the field of mechanobiology. The role of Piezo1 in stem cell guidance (Orapiriyakul et al. 2020), the non-invasive characterization of mechanical properties in 3D using Brillouin microscopy (Mattarelli et al. 2020), and the design of clinical cell analysis tools based on acoustofluidics (Mejía Morales et al. 2021) are some of the research lines that I expect to explore in the next years.

\section{Conclusions}

I interpret science as a random walk across questions and challenges, driven by personal curiosity and global goals. I consider collaboration the most powerful tool to orient the research in this forest of unknowns. I thank all the friends I had the opportunity to work with, and I hope to meet many more in the rest of my career.

\section{References}

Baldini, F., A. Bartolozzi, M. Ardito, A. Voci, P. Portincasa, M. Vassalli, and L. Vergani. 2019. "Biomechanics of cultured hepatic cells during different steatogenic hits." Journal of the Mechanical Behavior of Biomedical Materials 97. https://doi.org/10.1016/j.jmbbm.2019. 05.036 .

Bartolozzi A, Viti F, De Stefano S, Sbrana F, Petecchia L, Gavazzo P, Vassalli M (2020) Development of label-free biophysical markers in osteogenic maturation. Journal of the Mechanical Behavior of Biomedical Materials 103(March):103581. https://doi.org/10.1016/ j.jmbbm.2019.103581

Basso M, Paoletti P, Tiribilli B, Vassalli M (2008) Modelling and analysis of autonomous micro-cantilever oscillations. Nanotechnology 19(47):475501. https://doi.org/10.1088/0957-4484/19/47/475501

Basso M, Paoletti P, Tiribilli B, Vassalli M (2011) AFM imaging via nonlinear control of self-driven cantilever oscillations. IEEE Transactions on Nanotechnology 10(3):560-565. https://doi.org/ 10.1109/TNANO.2010.2051815

Best RB, Clarke J (2002) What can atomic force microscopy tell us about protein folding? Chemical Communications 3(January):183-192. https://doi.org/10.1039/b108159b

Bloise N, Petecchia L, Ceccarelli G, Fassina L, Usai C, Bertoglio F, Balli $M$ et al (2019) The effect of pulsed electromagnetic field exposure on osteoinduction of human mesenchymal stem cells cultured on nano-TiO \textlessinfltextgreater2 $\backslash$ textless/Infltextgreater Surfaces. PLoS ONE 13(6). https://doi.org/10.1371/journal.pone.0199046

Clarke L, Glendinning I, Hempel R (1994) The MPI message passing interface standard. In: Decker KM, Rehmann RM (eds) Programming Environments for Massively Parallel Distributed Systems. Birkhäuser Basel, Basel, pp 213-218. https://doi.org/10. 1007/978-3-0348-8534-8_21

Coste B, Mathur J, Schmidt M, Earley TJ, Ranade S, Petrus MJ, Dubin AE, Patapoutian A (2010) Piezo1 and Piezo2 are essential components of distinct mechanically activated cation channels. Science 330(6000):55-60. https://doi.org/10.1126/science.1193270

Crooks GE (1999) Entropy production fluctuation theorem and the nonequilibrium work relation for free energy differences. Physical Review E 60(3):2721-2726. https://doi.org/10.1103/PhysRevE.60. 2721

Crutzen, Paul J. 1995. "My Life with O3, NOx and Other YZOxs." Lecture presented at the Nobel Lecture. https://www.nobelprize. org/prizes/chemistry/1995/crutzen/lecture/.

Ferrari A, Capitanio M, Vassalli M, Martinac B (2019) Science by the sea: how nanoengineering met mechanobiology in Camogli. Biophysical Reviews 11(5):659-661. https://doi.org/10.1007/ s12551-019-00598-z

Galante D, Corsaro A, Florio T, Vella S, Pagano A, Sbrana F, Vassalli M, Perico A, D'Arrigo C (2012) Differential toxicity, conformation and morphology of typical initial aggregation states of $A \beta 1-42$ and A $\beta$ py3-42 beta-amyloids. International Journal of Biochemistry and Cell Biology 44(11):2085-2093. https://doi.org/10.1016/j. biocel.2012.08.010

Gavazzo P, Vassalli M, Costa D, Pagano A (2013) Novel NcRNAs Transcribed by Pol III and elucidation of their functional relevance by biophysical approaches. Frontiers in Cellular Neuroscience 7(203):1-6. https://doi.org/10.3389/fncel.2013.00203

Giacomelli G, Hegger R, Politi A, Vassalli M (2000) Convective Lyapunov exponents and propagation of correlations. Physical Review Letters 85(17):3616-3619. https://doi.org/10.1103/ PhysRevLett.85.3616

Giardina C, Livi R, Politi A, Vassalli M (2000) Finite thermal conductivity in 1D lattices. Physical Review Letters 84(10):2144-2147. https://doi.org/10.1103/PhysRevLett.84.2144 
Helenius J, Heisenberg C-P, Gaub HE, Muller DJ (2008) Single-cell force spectroscopy. Journal of Cell Science 121(11):1785-1791. https:// doi.org/10.1242/jcs.030999

Imparato A, Sbrana F, Vassalli M (2008) Reconstructing the free-energy landscape of a polyprotein by single-molecule experiments. EPL (Europhysics Letters) 82(5):58006. https://doi.org/10.1209/02955075/82/58006

Jarzynski C (1997) Nonequilibrium equality for free energy differences. Physical Review Letters 78(14):2690-2693. https://doi.org/10.1103/ PhysRevLett.78.2690

Liphardt J (2002) Equilibrium information from nonequilibrium measurements in an experimental test of Jarzynski's equality. Science 296(5574):1832-1835. https://doi.org/10.1126/science.1071152

Marchetti S, Sbrana F, Toscano A, Fratini E, Carlà M, Vassalli M, Tiribilli B, Pacini A, Gambi CMC (2011) $\beta$-Connectin Studies by small-angle $\mathrm{x}$-ray scattering and single-molecule force spectroscopy by atomic force microscopy. Physical Review E - Statistical, Nonlinear, and Soft Matter Physics 83(5). https://doi.org/10.1103/ PhysRevE.83.051919

Mattarelli M, Vassalli M, Caponi S (2020) Relevant length scales in brillouin imaging of biomaterials: the interplay between phonons propagation and light focalization. ACS Photonics 7(9). https://doi. org/10.1021/acsphotonics.0c00801

Mescola A, Vella S, Scotto M, Gavazzo P, Canale C, Diaspro A, Pagano A, Vassalli M (2012) Probing cytoskeleton organisation of neuroblastoma cells with single-cell force spectroscopy. Journal of Molecular Recognition 25(5):270-277. https://doi.org/10.1002/ jmr. 2173

Monaldi I, Vassalli M, Bachi A, Giovedì S, Millo E, Valtorta F, Raiteri R, Benfenati F, Fassio A (2010) The highly conserved synapsin domain e mediates synapsin dimerization and phospholipid vesicle clustering. Biochemical Journal 426(1):55-64. https://doi.org/10. 1042/BJ20090762

Morales M, Julián BH, Lippi GL, Vassalli M, Glynne-Jones P (2021) Acoustofluidic phase microscopy in a tilted segmentation-free configuration. Biomicrofluidics 15(1):014102. https://doi.org/10.1063/ 5.0036585

Orapiriyakul W, Tsimbouri MP, Childs P, Campsie P, Wells J, Fernandez-Yague MA, Burgess K et al (2020) Nanovibrational stimulation of mesenchymal stem cells induces therapeutic reactive oxygen species and inflammation for three-dimensional bone tissue engineering. ACS Nano 14(8):10027-10044. https://doi.org/10. 1021/acsnano.0c03130

Paoletti P, Basso M, Pini V, Tiribilli B, Vassalli M (2011) Self-driven soft imaging in liquid by means of photothermal excitation. Journal of Applied Physics 110(11). https://doi.org/10.1063/1.3665396

Papi M, Arcovito G, De Spirito M, Vassalli M, Tiribilli B (2006) Fluid viscosity determination by means of uncalibrated atomic force microscopy cantilevers. Applied Physics Letters 88(19). https://doi.org/ $10.1063 / 1.2200588$

Papi M, Maulucci G, Arcovito G, Paoletti P, Vassalli M, De Spirito M (2008) Detection of microviscosity by using uncalibrated atomic force microscopy cantilevers. Applied Physics Letters 93(12). https://doi.org/10.1063/1.2970963

Petecchia L, Sbrana F, Utzeri R, Vercellino M, Usai C, Visai L, Vassalli M, Gavazzo P (2015) Electro-magnetic field promotes osteogenic differentiation of bm-hmscs through a selective action on ca2+-related mechanisms. Scientific Reports 5(1):13856. https://doi.org/10. 1038/srep 13856

Pini V, Tiribilli B, Gambi CMC, Vassalli M (2010) Dynamical characterization of vibrating afm cantilevers forced by photothermal excitation. Physical Review B - Condensed Matter and Materials Physics 81(5). https://doi.org/10.1103/PhysRevB.81.054302

Ridone P, Pandzic E, Vassalli M, Cox CD, Macmillan A, Gottlieb PA, Martinac B (2020) Disruption of membrane cholesterol organization impairs the activity of PIEZO1 channel clusters. Journal of General Physiology 152(8):e201912515. https://doi.org/10.1085/jgp. 201912515

Ridone P, Vassalli M, Martinac B (2019) Piezo1 mechanosensitive channels: what are they and why are they important. Biophysical Reviews 11(5):795-805. https://doi.org/10.1007/s12551-019-00584-5

Rief M (1997) Reversible unfolding of individual titin immunoglobulin domains by AFM. Science 276(5315):1109-1112. https://doi.org/ 10.1126/science.276.5315.1109

Righini G (1967) The Arcetri astrophysical observatory. Solar Physics 1(3-4):494-497. https://doi.org/10.1007/BF00151376

Sbrana F, Fotia C, Bracalello A, Baldini N, Marletta G, Ciapetti G, Bochicchio B, Vassalli M (2012) Multiscale characterization of a chimeric biomimetic polypeptide for stem cell culture. Bioinspiration \& Biomimetics 7(4):046007. https://doi.org/10. 1088/1748-3182/7/4/046007

Sbrana F, Lorusso M, Canale C, Bochicchio B, Vassalli M (2011) Effect of chemical cross-linking on the mechanical properties of elastomeric peptides studied by single molecule force spectroscopy. Journal of Biomechanics 44(11):2118-2122. https://doi.org/10.1016/j. jbiomech.2011.05.028

Sheetz M, Hanry Y (2018) The cell as a machine: 1st ed. Cambridge University Press. https://doi.org/10.1017/9781107280809

Tatham AS, Shewry PR (2000) Elastomeric proteins: biological roles, structures and mechanisms. Trends in Biochemical Sciences 25(11):567-571. https://doi.org/10.1016/S0968-0004(00)01670-4

Thellung S, Gatta E, Pellistri F, Corsaro A, Villa V, Vassalli M, Robello M, Florio T (2013) Excitotoxicity through NMDA receptors mediates cerebellar granule neuron apoptosis induced by prion protein 90-231 fragment. Neurotoxicity Research 23(4):301-314. https:// doi.org/10.1007/s12640-012-9340-9

Tsushima H, Emanuele M, Polenghi A, Esposito A, Vassalli M, Barberis A, Difato F, Chieregatti E (2015) HDAC6 and RhoA are novel players in abeta-driven disruption of neuronal polarity. Nature Communications 6:7781. https://doi.org/10.1038/ncomms8781

Vassalli M, Pini V, Tiribilli B (2010) Role of the driving laser position on atomic force microscopy cantilevers excited by photothermal and radiation pressure effects. Applied Physics Letters 97(14). https:// doi.org/10.1063/1.3497074

Vassalli M, Sbrana F, Laurita A, Papi M, Bloise N, Visai L, Bochicchio B (2013) Biological and structural characterization of a naturally inspired material engineered from elastin as a candidate for tissue engineering applications. Langmuir 29(51):15898-15906. https:// doi.org/10.1021/la403311x

Zanghirati, G., F. Cocco, F. Taddei, and G. Paruolo. 1999. “Cray T3E performances of a parallel code for a stochastic dynamic assets and liabilities management model $\star$." In Euro-Par'99 Parallel Processing, edited by Patrick Amestoy, Philippe Berger, Michel Daydé, Daniel Ruiz, Iain Duff, Valérie Frayssé, and Luc Giraud, 1685:1176-86. Lecture notes in computer science. Berlin, Heidelberg: Springer Berlin Heidelberg. https://doi.org/10.1007/3540-48311-X_166.

Publisher's note Springer Nature remains neutral with regard to jurisdictional claims in published maps and institutional affiliations. 